\begin{tabular}{|c|c|c|c|c|c|}
\hline MUNIBE Antropologia-Arkeologia & $n^{\circ} 68$ & $73-84$ & DONOSTIA & 2017 & ISSN 1132-2217 • elSSN 2172-4555 \\
\hline
\end{tabular}

\title{
Aves de caza. Estudio tafonómico y zooarqueológico de los restos avianos de los niveles musterienses de Pié Lombard (Alpes-Maritimes, Francia)
}

\author{
Hunting birds. Taphonomy and Zooarchaeological analysis of avian \\ remains of Mousterian levels of Pié Lombard (Alpes-Maritimes, France)
}

PALABRAS CLAVES: Tafonomía, zooarqueología, huesos avianos, explotación de aves, pequeñas presas, neandertales, rapaces, pequeños carnívoros.

GAKO-HITZAK: Tafonomia, zooarkeologia, hezur abianoak, hegaztien ustiapena, harrapakin txikiak, neandertalak, harrapariak, karniboro txikiak. KEY WORDS: Taphonomy, zooarchaeology, avian bones, exploitation of birds, small game, Neanderthals, raptors, small carnivores.

\section{A.J. ROMERO(1), J. C. DÍEZ(2) y J.P. BRUGAL (3)}

\section{RESUMEN}

El yacimiento musteriense de Pié Lombard (Alpes-Maritimes, Francia) está localizado en un entorno montañoso de medio rango y posee un conjunto óseo rico y diversificado en el cual los restos de aves son abundantes. Su estudio tafonómico y zooarqueológico brinda cuestiones importantes sobre la explotación de aves por parte de rapaces, carnívoros y neandertales, y añade otro yacimiento en el cual se lleva a cabo la explotación de pequeñas presas en Europa Occidental.

\section{LABURPENA}

Pié Lombard-eko (Itsas Alpeak, Frantzia) aztarnategi mousteriarra maila ertaineko ingurune menditsuan dago, eta hezur-multzo aberatsa eta dibertsifikatua du. Oso ugariak dira hegaztien hondakinak. Azterketa tafonomikoak eta zooarkeologikoak harrapariek, karniboroek eta neandertalek hegaztiekin lotuta egindako ustiapenari buruzko gai esanguratsuak azaleratu ditu. Gainera, mendebaldeko Europan harrapakin txikien ustiapena egin deneko beste aztarnategi bat azaldu da.

\section{ABSTRACT}

Traditionally small fast game exploitation is characteristic of the end of Upper Palaeolithic or modern humans. Nevertheless, several authors demonstrated recently small fast game exploitation by fossil hominins able to capture, process and consume small animals for different purposes, despite numerous methodological and technical problems. In this work, we provide results of a taphonomical and zooarchaeological study on all bird bones from the Middle Palaeolithic levels of Pié Lombard using various analytical tools.

The Mousterian site of Pié Lombard is a narrow rock shelter located in a middle-range mountainous environment near Tourrettes-sur-Loup (Alpes-Maritimes, France). The Middle Palaeolithic bird bone assemblage retained 18 taxa, all adults. The most represented taxa are choughs and pigeons that represent rocky species, characteristic of mild-temperate climate. The third most common specie is the partridge, although to a lesser extent. Long bones are predominant and anatomical representations are different between the frequent species. Various taphonomical alterations are visible on bird remains, including human modifications (cut marks -incisions and scraping marks- and burnt bones), other non-human predator activities (usually tooth marks and digestion processes) and some diagenetic alterations (especially concretion, corrosion and root marks). The majority of the bones are characterized by curved outline, mixed angles and jagged textures.

The bird remains exhibit significant breakages due to various consumption processes (humans, raptors and small carnivores) completed by natural burial process. The birds of prey (probably nocturnal raptors) transported their prey (usually choughs) to the rock shelter where they were consumed and regurgitated. Secondly, small and medium-sized carnivores (small felids like lynx or wild cat had a relevant role) predate occasionally on birds. Neanderthals occupations were occasional, where they exploited herbivores but are also in to some extent at the source of bird accumulation. The humans captured almost exclusively pigeons and partridges, and some choughs. Without excluding a possible use of feathers, the Neanderthal groups of Pié Lombard acquired birds for their meat.

Thus, Pié Lombard is a new Middle Paleolithic site where small game exploitation was a real part of the diet. The site can be is added to our recent knowledge, as another sites in Western Europe, demonstrating the ability of Neanderthal to hunt and consume birds as a continuous economic activity in space and time.

\footnotetext{
(1) Universidad del País Vasco (UPV/EHU), Departamento de Geografía, Prehistoria y Arqueología, C/Tomás y Valiente, s/n, C.P.: 01006, Vitoria-Gasteiz, España, antoniojesus.romero@ehu.eus

(2) Universidad de Burgos (UBU), Departamento de Historia, Geografía y Comunicación, Laboratorio de Prehistoria I+D+i, clomana@ubu.es

(3) CNRS, USR 3336, IFRA, Nairobi, Kenya \& AMU, UMR 7269 LAMPEA, Aix-en-Provence, France, brugal@mmsh.univ-aix.fr
} 


\section{1.- INTRODUCCIÓN}

\subsection{Estado de la cuestión}

Tradicionalmente, la explotación del small fast game (en términos de Cochard et al., 2012) es característica del final del Paleolítico Superior (p.e. Díez et al., 1995, Cassoli and Tagliacozzo, 1997, Eastham, 1998, Laroulandie, 2003, 2005a, Fa et al., 2013) o, en cualquier caso, de nuestra especie (p.e. Val et al., 2016).

Sin embargo, en los últimos años no pocos autores han demostrado la adquisición, procesado y aprovechamiento de aves y lagomorfos por parte de homininos fósiles con diferentes propósitos (p.e., Sanchis and Fernández-Peris, 2008, Cochard et al., 2012, Sanchis, 2012, Blasco et al., 2016, Laroulandie et al., 2016, Romandini et al., 2016). Así, este paradigma clásico ha sido sobrepasado, especialmente para el caso de las sociedades neandertales.

No obstante, en arqueología prehistórica, encontramos numerosos problemas metodológicos y técnicos para detectar un aprovechamiento de las pequeñas presas en sociedades del Pleistoceno:

- Es frecuente que los yacimientos del Pleistoceno sean palimpsestos (Bailey, 2007). En ellos resulta complejo manejar las escalas temporales (Vaquero, 2008) y conocer con detalle cómo estas afectan a los procesos de formación del depósito arqueológico en cuestión.

- La recuperación de elementos anatómicos de pequeños animales (como lagomorfos, roedores, aves o reptiles) presenta complicaciones técnicas, y en pocas ocasiones pueden hallarse in situ en contextos arqueológicos claros.

- La presencia de carcasas de pequeños animales en yacimientos arqueopaleontológicos suele deberse a distintas causas (p.e., Laroulandie et al., 2016, Rufà et al., 2016a, 2016b) y no siempre es posible conocer exactamente la intervención de cada uno de los agentes tafonómicos y su grado de actuación.

- Los restos de animales pequeños suelen tener características anatómicas diferentes a los de animales de tallas medias y grandes y no siempre son bien conocidas en la literatura científica. Por ello, su análisis tafonómico no suele ser sencillo. En suma, los procesos de consumo pueden causar la destrucción de algunos elementos, dificultando aún más la recuperación arqueológica de los mismos.

- Por estas razones, los referentes etnográficos (p.e., Landt, 2007, Martínez, 2009, Negro et al., 2016) y experimentales (Andrews, 1995, Laroulandie, 2005b, Lloveras et al., 2009, Sanchis et al., 2011, Romandini et al., 2016, Pedergnana y Blasco, 2016, Romero et al., 2016a) resultan relevantes para el estudio y la resolución de problemas arqueológicos en este sentido.

En este trabajo realizamos un estudio tafonómico y zooarqueológico de todos los restos avianos de los niveles del Paleolítico Medio del yacimiento de Pié Lom- bard (Tourrettes-sur-Loup, Alpes-Maritimes, Francia) empleando todas las herramientas metodológicas disponibles en la actualidad. El objetivo es el de comprender el papel de estos restos de aves en este sitio Musteriense, así como conocer los agentes tafonómicos involucrados (humanos, carnívoros, rapaces u otros).

\subsection{El yacimiento de Pié Lombard}

Pié Lombard es un pequeño abrigo rocoso cerca de Tourrettes-sur-Loup (Alpes-Maritimes, Francia). Se encuentra a $9 \mathrm{~km}$ del Mediterráneo y a $15 \mathrm{~km}$ al sur de la falda de los Alpes. El enclave está situado a $250 \mathrm{~m}$ de altitud (50 metros sobre el río Loup, el cual ha generado unas espectaculares gargantas en calizas jurásicas). Las excavaciones arqueológicas se desarrollaron en los años setenta y ochenta del siglo pasado (Texier et al., 2011). Estos trabajos recuperaron una importante cantidad de restos óseos acumulados en una diaclasa en la parte delantera del abrigo. La fosa, de unos 10 m3, funciona como trampa sedimentaria, ya que el resto del abrigo apenas ha conservado material arqueopaleontológico. Esta secuencia, de unos $3 \mathrm{~m}$ de espesor, se subdivide en varias capas (Texier et al., 2011) y en dos complejos principales.

Así, existen dos complejos estratigráficos: el superior corresponde al Paleolítico Superior o al Epipaleolítico (no existe consenso al respecto); el inferior se adscribe al Musteriense. El complejo musteriense posee un espesor medio de $1 \mathrm{~m}$ de espesura y está formado por una matriz arcillo arenosa parda y marrón, dividido en cinco tallas que no pudieron validarse en los estudios de laboratorio (Porraz, 2005). Parece probable que la mayor parte del depósito fuese acumulada en una franja corta de tiempo a partir de su desplazamiento desde el abrigo rocoso. Las dataciones por TL arrojan fechas en torno a los 70.000 años de antigüedad (transición entre los estadios isotópicos 4 y 5) (Valladas et al., 1987). Los sedimentos se apoyan en una costra estalagmítica datada por ESR entre $147 \pm 10$ ka y $130 \pm 20$ ka (Yokoyama et al., 1983).

El conjunto de industria lítica $(n=902)$ ha sido adscrito claramente al Musteriense. Bastantes piezas líticas están retocadas y manufacturadas con la técnica Levallois. La materia prima más empleada es local. Sin embargo, se ha detectado el uso esporádico de microcuarcitas y jaspes, los cuales proceden de 60 y $240 \mathrm{~km}$ respectivamente (Porraz, 2005). Es muy probable que la industria fuera realizada por comunidades neandertales, a juzgar por la presencia de dos restos dentarios de un niño adscritos a dicha especie (Texier et al., 2011).

En relación a la fauna, hallamos entre los grandes mamíferos 15 especies en toda la secuencia (7 carnívoros y 8 herbívoros) a partir de 4686 elementos identificables (determinables y/o clasificables por tallas). Los carnívoros representan el 8,4\% de los restos, siendo el leopardo y el lobo los taxones más abundantes se- 
guidos del lince. Los carnívoros de pequeño tamaño, como el zorro y el gato silvestre, están presentes junto con el oso. Por contra, la presencia del cuón es extraña. Los predadores están representados por individuos de todas las edades (jóvenes, adultos o viejos). Todo indica que ocupaban el sitio durante periodos prolongados de forma exclusiva, reproduciéndose, hibernando o falleciendo en el entorno.

La cabra montés y el ciervo rojo representan el $38,6 \%$ y el $35 \%$ de los restos, respectivamente, siendo frecuentes tantos los juveniles como los adultos. Otros herbívoros presentes son el rebeco, el uro, el corzo, el gamo y el jabalí. La presencia de elefante (Palaeoloxodon cf. antiquus) se constata a partir de un fragmento de colmillo, como en otro yacimiento del Paleolítico Medio en el Sureste francés (Abrigo rocoso de Mandrin, Drôme; Brugal, e. p.). Finalmente, los lepóridos (conejo) están presentes con ca. 1300 restos y, como mínimo, 23 individuos, la mayoría adultos (Gerber, 1973). Además, dos dientes humanos aislados (incisivos deciduales) son atribuidos a un joven individuo neandertal, con entre 2 y 4 años de edad (Texier et al., 2011). También hallamos microfauna, registrando 2 especies de insectívoros, 5 quirópteros, 1 anfibio, 2 reptiles y 12 roedores (incluyendo castor y marmota alpina) y 18 taxones de aves (Texier et al., 2011).

A partir de la información malacológica y palinológica, el paleoambiente de este periodo indica zonas boscosas y abiertas de un biotopo de altura de condiciones más frías que las actuales aunque marcado por una influencia mediterránea.

\section{MATERIALES Y MÉTODOS}

En este trabajo estudiamos todos los restos de aves (1970 NR, 1680 NISP) del paquete estratigráfico musteriense (Complejo II) del yacimiento de Pié Lombard (Tourrettes-sur-Loup). Este material se conserva en el Laboratoire Méditerranéen de Préhistoire (Aix-en-Provence, Francia) y fue recuperado bajo la dirección de P.-J. Texier (Université Bordeaux 1) entre 1971 y 1996. Durante los trabajos arqueológicos, P.-J. Texier y E. Desclaux separaron los restos de aves de otros materiales.

Procesamos todos los restos avianos como un único nivel (Complejo II) para obtener una visión general y poder obviar algunos problemas estratigráficos. El análisis fue realizado siguiendo los principios generales de la tafonomía (p. e., Lyman, 1994, 2008).

La mayoría de los restos óseos aquí presentados poseían una clasificación taxonómica realizada por $\mathrm{C}$. Mourer-Chauviré (1975) (por especies, género o grupo, dependiendo de las posibilidades). En algunas ocasiones hemos trabajado con especies o géneros (sobre todo en el caso de Pyrrhocorax). Además, extrajimos del conjunto de aves datos paleogeográficos y paleoecológicos (Sánchez-Marco, 1996, 2004).
En primer lugar, identificamos cada resto tanto anatómica como taxonómicamente. Asimismo, indicamos el NISP de cada especie o grupo, a la vez que calculamos el NME y el NMI a partir de los elementos anatómicos y su lateralización. En segundo lugar, anotamos en cada caso una edad aproximada (inmadura o adulta) empleando la relativa porosidad de los huesos (Serjeantson, 2009). Al mismo tiempo, calculamos una serie de ratios: a) wing/leg, calculado dividiendo el número total de restos del ala (carpometacarpo, ulna y húmero) entre la suma total de restos de alas y patas (tarsometatarso, tibiotarso y fémur) (Ericson, 1987, Livingston, 1989); b) core/limb, calculado dividiendo el total de elementos del "núcleo" del ave (esternón, coracoides, escápula y pelvis) entre la suma total de elementos del núcleo y las extremidades (carpometacarpo, ulna, radio, húmero, fémur, tibiotarso y tarsometatarso) (Branwell et al., 1987, Bochenski, 2005); c) proximal/distal, calculado dividiendo los elementos proximales (coracoides, escápula, húmero, fémur y tibiotarso) entre la suma total de elementos proximales y distales (carpometacarpo, ulna, radio y tarsometatarso) (Bochenski, 2001). Finalmente, empleamos una prueba de ji-cuadrado (x2) para evaluar la significancia y la relación entre las diferentes variables aquí comentadas.

En relación con el análisis tafonómico, en primer lugar prestamos atención a la integridad de cada resto óseo, indicando si estaba completo o fragmentado. En segundo lugar, observamos todas las superficies óseas con una lupa binocular Nikon SMZ 1500 7.5-125x y un Dino-Lite Pro AM 413ZT registrando todas las alteraciones.

Tuvimos en cuenta tanto las alteraciones diagenéticas (marcas de raíces, corrosión, concreción, fisurados, impregnaciones de manganeso y óxidos) como las modificaciones bioestratinómicas (marcas de dientes, incluyendo primarias -pits, punctures y scores- y secundarias -furrowing, bordes crenulados y crushing-, marcas de roedores y huellas de digestión). Para estas últimas, elaboramos una clasificación con cuatro niveles (0- no digerido; 1- light; 2- moderado; 3- heavy) partiendo de los trabajos de varios autores (Andrews, 1990, Lloveras et al., 2008, 2012). Por otro lado, también registramos intervenciones antrópicas (marcas de corte -siguiendo la clasificación de Noe-Nygaard (1989): incisiones, tajos, raspados y aserrados-, huesos quemados y algunas fracturas y daños producidos con los dientes exclusivamente por parte de humanos, como peeling o squashing (Laroulandie, 2005b). Con respecto a los huesos quemados, elaboramos otra tipología inspirados por trabajos clásicos sobre esta materia (p. e., Shipman et al., 1984, Nicholson, 1993, Stiner y Kuhn, 1995) con cinco niveles según el color y las alteraciones ocasionadas por la cremación: 0- no quemado; 1- marrón; 2- negro; 3- gris y 4- blanco.

Además, evaluamos la fracturación existente en el conjunto aquí analizado con el fin de poder conocer las causas de la fragmentación. Así, intentamos diferenciar entre fracturas producidas en fresco y en seco, ponien- 
do atención a un posible origen antrópico. Así, en relación a otros estudios (Villa and Mahieu, 1991, Romero et al., 2016a) anotamos las siguientes variables (cuando ello fue posible) sobre cada fractura: a) delineación (transversal o curva); b) ángulo (oblicuo, recto o mixto); c) textura (suave o dentada) (Villa and Mahieu, 1991). Adicionalmente, incluimos una clasificación morfológica (Romero et al., 2016a): a) transversal, subdividido en transversal simple (T1), transversal columnar (T2), transversal con piso (T3), irregular transversal (T4), transversal con uno (TL1), dos (TL2) o más escalones (TL3); b) curvo, subdividido en curvo simple (C1), curvo columnar (C2) y curvo con pico (C3); c) longitudinal (L) y transversal curvo (TC).

\section{RESULTADOS}

Constatamos la presencia de 18 taxones de aves (Passeriformes indet. del análisis de Mourer-Chauviré incluidos) (Tabla I), todas adultas. Los grupos más representados son las chovas (Pyrrhocorax pyrrhocorax, Pyrrhocorax graculus y Pyrrhocorax sp.), las palomas (Columba livia) y, a mucha distancia, las perdices (sobre todo, Alectoris graeca). Las especies más comunes en el yacimiento son características de zonas de roquedo y propias de climas templados (Sánchez-Marco, 1996).

Los huesos largos son los más comunes en el conjunto (Figura 1). No obstante, la presencia de huesos articulares, falanges y otros huesos pequeños es indi-

\begin{tabular}{|l|c|c|c|c|c|c|}
\hline \multicolumn{1}{|c|}{ Species } & NISP & \%NISP & MNE & \%MNE & MNI & \%MNI \\
\hline Alectoris graeca & 26 & $1,54 \%$ & 21 & $2,29 \%$ & 3 & $2,65 \%$ \\
\hline Anas platyrhynchos & 2 & $0,12 \%$ & 2 & $0,22 \%$ & 1 & $0,88 \%$ \\
\hline Anthus cf. campestris & 1 & $0,06 \%$ & 1 & $0,11 \%$ & 1 & $0,88 \%$ \\
\hline Aquila chrysaetos & 4 & $0,24 \%$ & 4 & $0,44 \%$ & 1 & $0,88 \%$ \\
\hline Carduelis carduelis & 1 & $0,06 \%$ & 1 & $0,11 \%$ & 1 & $0,88 \%$ \\
\hline C. coccothraustes & 2 & $0,12 \%$ & 2 & $0,22 \%$ & 1 & $0,88 \%$ \\
\hline Columba livia & 348 & $20,65 \%$ & 191 & $20,81 \%$ & 23 & $20,35 \%$ \\
\hline Corvus corax & 5 & $0,30 \%$ & 5 & $0,54 \%$ & 1 & $0,88 \%$ \\
\hline Corvus cf. corone & 2 & $0,12 \%$ & 2 & $0,22 \%$ & 1 & $0,88 \%$ \\
\hline G. garrulus & 3 & $0,18 \%$ & 3 & $0,33 \%$ & 1 & $0,88 \%$ \\
\hline Hirundo rustica & 2 & $0,12 \%$ & 1 & $0,11 \%$ & 1 & $0,88 \%$ \\
\hline Perdix perdix & 13 & $0,77 \%$ & 12 & $1,31 \%$ & 2 & $1,77 \%$ \\
\hline Ptyonoprogne rupestris & 5 & $0,30 \%$ & 4 & $0,44 \%$ & 3 & $2,65 \%$ \\
\hline P. pyrrhocorax & 111 & $6,59 \%$ & 83 & $9,04 \%$ & 10 & $8,85 \%$ \\
\hline P. graculus & 304 & $18,04 \%$ & 138 & $15,03 \%$ & 31 & $27,43 \%$ \\
\hline Pyrrhocorax sp. & 676 & $40,12 \%$ & 446 & $48,58 \%$ & 32 & $28,32 \%$ \\
\hline Turdus merula & 2 & $0,12 \%$ & 2 & $0,22 \%$ & 1 & $0,88 \%$ \\
\hline Passeriformes & 178 & $10,56 \%$ & 0 & $0,00 \%$ & 0 & $0,00 \%$ \\
\hline Total & $\mathbf{1 6 8 5}$ & $\mathbf{1 0 0} \%$ & $\mathbf{9 1 8}$ & $\mathbf{1 0 0} \%$ & $\mathbf{1 1 3}$ & $\mathbf{1 0 0} \%$ \\
\hline
\end{tabular}

Tabla 1: Representación de las especies del yacimiento de Pié Lombard. / Representation of species in Pié Lombard site.

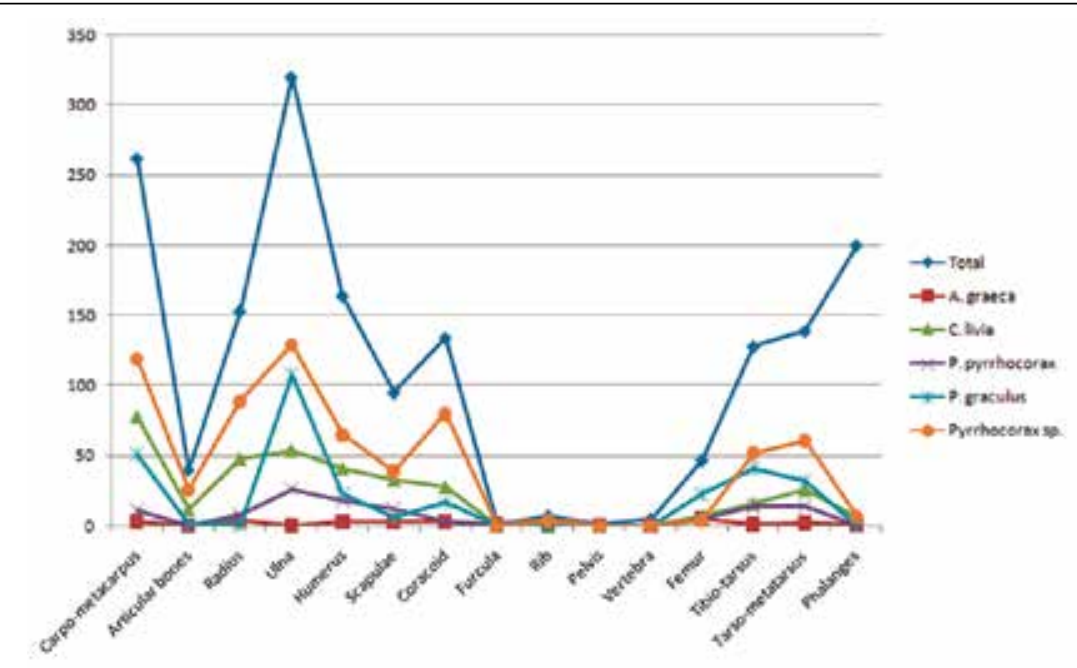

Fig. 1. Elementos anatómicos (NISP) de Pié Lombard. Total y especies principales. / Anatomic elements (NISP) of Pié Lombard. Total and principal species. 
cativa de unas buenas técnicas de excavación y tratamiento de los sedimentos. En términos generales, los elementos más importantes de estas especies son los huesos de las alas (Tabla II). Sin embargo, existe una representación anatómica diferencial entre las especies más frecuentes del sitio (Alectoris graeca, Columba livia, Pyrrhocorax pyrrhocorax, Pyrrhocorax gracuIus y Pyrrhocorax sp.). Las extremidades en el género Pyrrhocorax (alas y patas) son los elementos mejor representados, al contrario que en los géneros Columba y Alectoris, que poseen una mayor representación de los huesos del "núcleo" (core en inglés) sobre los de las patas (Núcleo: 19,21\% en Alectoris graeca y Columba livia; $14,94 \%$ en Pyrrhocorax; Patas: $16,10 \%$ en Alectoris graeca y Columba livia; $23,41 \%$ en Pyrrhocorax). Esta distribución es estadísticamente significativa $\left(x^{2}=9.958 ; p=0.0068\right)$.

En este orden de cosas, algunas alteraciones tafonómicas son visibles sobre los restos avianos. Primero, sobre las intervenciones antrópicas, registramos marcas de corte en el 2,99\% $(n=59)$ de los huesos y un 1,47\% ( $n=29)$ están quemados (grado 1: $11 \mathrm{NR}$; grado 2: 13 NR; grado 3: 1 NR; grado 4: 2 NR) (Figura 2a).
De entre los huesos con marcas de corte, la mayoría de ellos presentan incisiones $(94,92 \%, n=56)$ frente a algunos raspados $(10,17 \%, n=6)$ (Figura $2 b$ ) (algunos combinados sobre los mismos restos). Los cortes suelen estar dispuestos, en primer lugar, oblicuos al eje longitudinal del hueso $(64,41 \%$ de los huesos con marcas de corte, $\mathrm{n}=38$ ) (Figura 2c). En segundo lugar, también hallamos un buen número de marcas de corte transversales $(59,32 \%, n=35)$ (Figura 2d). Por contra, la disposición longitudinal de estas marcas suele ser menos común $(6,78 \%, n=4)$. Las acciones reconocidas a partir de las características y la situación de las incisiones son el descarnado y/o la remoción de plumas $(81,36 \%, n=48)$ y, en menor medida, la desarticulación $(22,03 \%, n=13)$.

Las marcas de corte tienen una disposición similar en todas las especies manipuladas por los humanos. La mayoría se encuentran sobre los huesos de las alas $(69,49 \%$ de los huesos con incisiones antrópicas, $n=41)$, aunque también en elementos del núcleo $(20,34 \%, n=12)$ y de las patas $(10,17 \%, n=6)$. Esta distribución es estadísticamente significativa $(X 2=11.923$; $\mathrm{p}=0.0025)$. Por otro lado, la situación más común de los

\begin{tabular}{|l|c|c|c|c|c|c|c|c|c|}
\hline \multicolumn{1}{|c|}{ Especies } & Wings & Legs & Wings/Legs & Core & Limbs & Core/Limbs & Prox & Dist & Prox/Dist \\
\hline A. graeca & 6 & 8 & 42,86 & 7 & 18 & 28 & 15 & 9 & 62,5 \\
\hline C. livia & 173 & 57 & 75,22 & 61 & 270 & 18,43 & 125 & 206 & 37,76 \\
\hline P. pyrrhocorax & 55 & 33 & 62,5 & 15 & 96 & 13,51 & 52 & 59 & 46,85 \\
\hline P. graculus & 182 & 96 & 65,47 & 23 & 279 & 7,61 & 110 & 192 & 36,42 \\
\hline Pyrrhocorax sp. & 313 & 117 & 72,79 & 119 & 519 & 18,65 & 240 & 398 & 37,62 \\
\hline
\end{tabular}

Tabla 2: Ratios de elementos wing/leg, core/limb y proximal/distal de las especies más abundantes de Pié Lombard. / Wing/leg, core/limb and proximal/distal elements ratios in more abundant species in Pié Lombard.

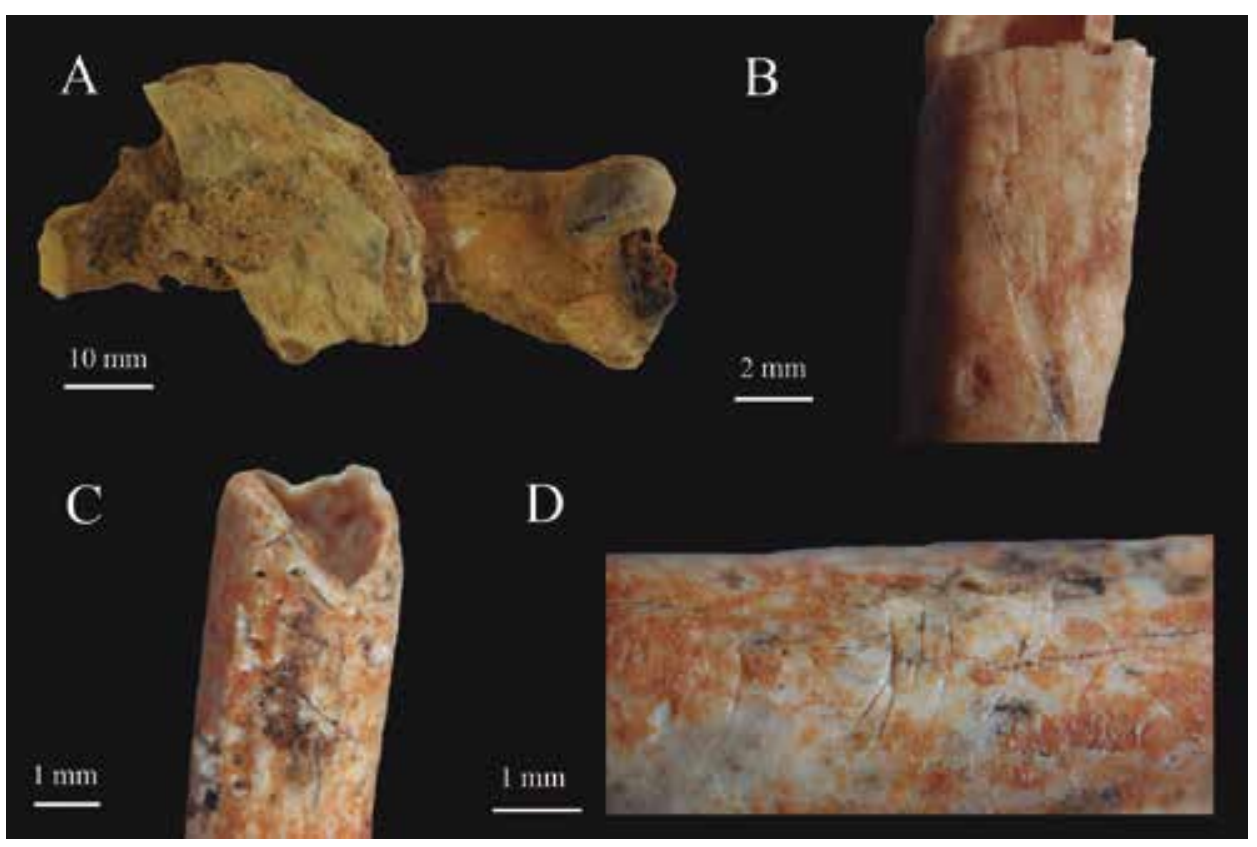

Fig. 2. Ejemplos de marcas de corte en el conjunto. A) Húmero distal (Columba livia) quemado; B) Húmero (Pyrrhocorax pyrrhocorax) con raspado longitudinal y un posible pit humano asociado; C) Radio (Columba livia) con marcas de corte oblicuas; D) Radio (Alectoris graeca) con marcas de corte transversal. / Examples of cutmarks in our sample. A) Distal humerus (Columba livia) burned; B) Humerus (Pyrrhocorax pyrrhocorax) with longitudinal scraping and a possible human pit associated; C) Radius (Columba livia) with oblique cutmarks; D) Radius (Alectoris graeca) with transversal cutmarks. 
cortes es la diáfisis $(66,10 \%, n=39)$, frente a las que se sitúan en las epífisis $(16,95 \%, n=10)$ o cerca de ellas $(16,95 \%, n=10)$.

Los grupos de aves con marcas de corte en Pié Lombard fueron desigualmente manipuladas por los humanos. Las especies con mayor interés por parte de los grupos de homininos en Pié Lombard (en relación con el número de NISP de cada especie) fueron, en primer lugar, Alectoris graeca $(23,08 \%$ de NISP de esta especie con incisiones antrópicas, $n=6)$, Columba livia $(6,57 \%, n=23)$ y el género Pyrrhocorax $(2,38 \%$, $\mathrm{n}=26)$. Esta distribución es estadísticamente significativa (x2=40.392; $p<0.0001)$.

Además, hallamos otras actividades de depredadores no humanos sobre los restos avianos. Encontramos pits y punctures sobre el $6,24 \%$ de los restos $(n=123)$ y scores en el 6,55\% ( $n=129)$. Por el contrario, otras alteraciones derivadas de la masticación son mucho más escasas, como el furrowing $(1,06 \%, n=21)$, los bordes crenulados $(0,91 \%, n=18)$, crushing $(0,15 \%, n=3)$, peeling $(0,15 \%, n=3)$ o marcas de roedores $(0,35 \%, n=7)$. Sin embargo, los procesos digestivos son bastante abundantes, estando presentes en más de una cuarta parte de la colección ósea $(25,58 \%, n=504)$ (Figura $3 a, b, d, e)$. Los procesos digestivos son más usuales sobre los restos del género Pyrrhocorax que en otras especies (Tabla III). En suma, la digestión con grado light es la más común en todos los restos.

Finalmente, también existen varias alteraciones diagenéticas sobre el conjunto aviano aquí presentado. La alteración diagenética más frecuente es la concreción (18,78\%, n=370). Asimismo, encontramos corrosión

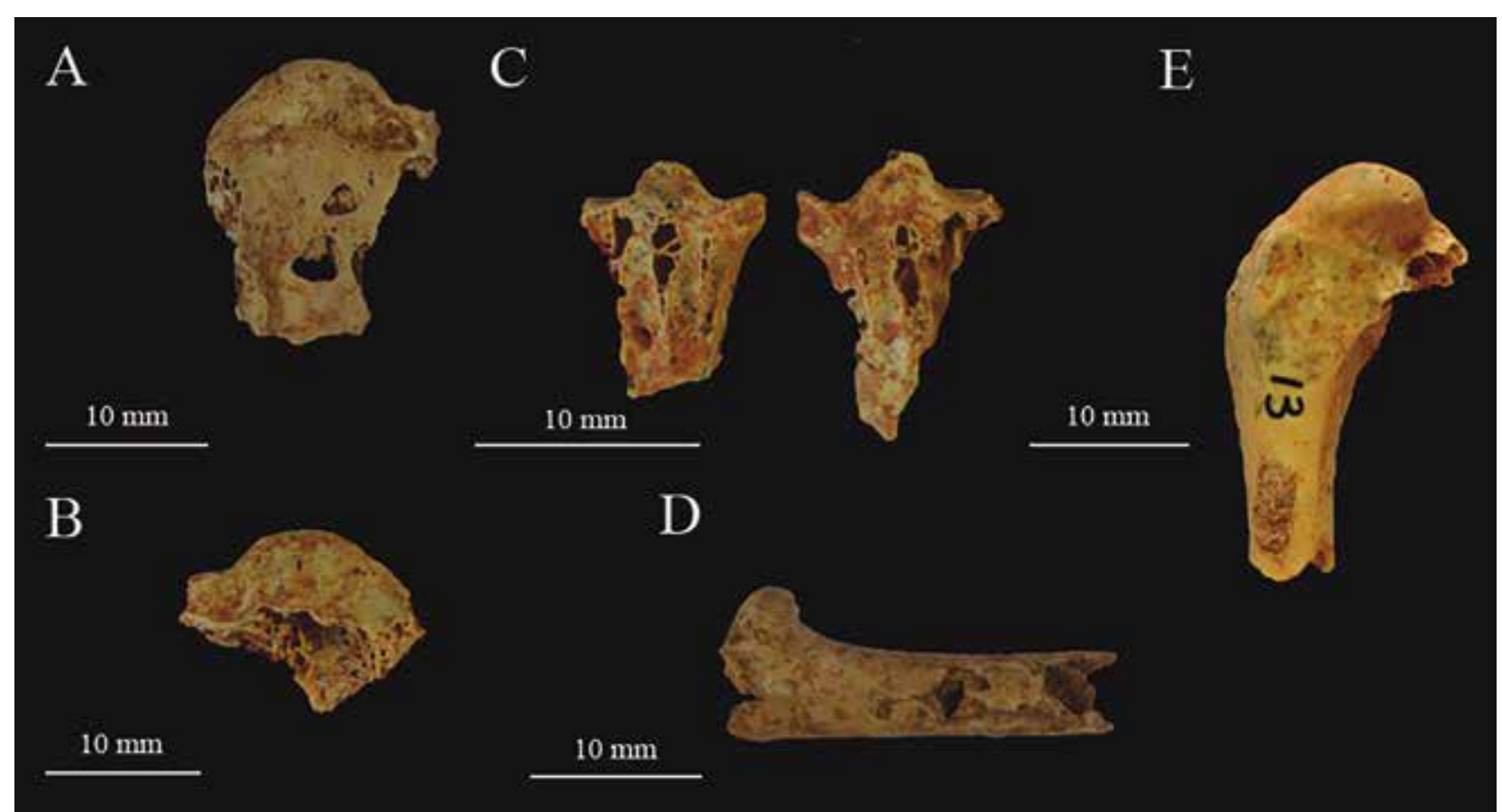

Fig. 3. Ejemplos de marcas de corte en nuestra muestra. A) Húmero proximal (Pyrrhocorax indet.) con punctures y bordes crenulados; B) Húmero proximal (Pyrrhocorax indet.) con bordes crenulados; C) Tarsometatarso proximal (Pyrrhocorax indet.) con procesos de digestión heavy; D) Ulna distal (Pyrrhocorax indet.) con pits, scores y punctures; E) Húmero proximal (Columba livia) con furrowing. / Examples of tooth marks in our sample. A) Proximal humerus (Pyrrhocorax indet.) with punctures and crenulated edges; B) Proximal humerus (Pyrrhocorax indet.) with crenulated edges; C) Proximal tarso-metatarsus (Pyrrhocorax indet.) with heavy digestion processes; D) Distal ulna (Pyrrhocorax indet.) with pits, scores and punctures; E) Proximal humerus (Columba livia) with furrowing.

\begin{tabular}{|l|c|c|c|c|c|c|c|c|c|c|}
\hline & A. graeca & \%A. graeca & C. livia & \%C. livia & P. pyr. & \%P. pyr. & P. grac. & \%P. grac. & P. indet & \%P. indet \\
\hline No digerido & 26 & $100,00 \%$ & 269 & $77,30 \%$ & 78 & $70,27 \%$ & 211 & $69,41 \%$ & 474 & $70,12 \%$ \\
\hline Digerido & 0 & $0,00 \%$ & 79 & $22,70 \%$ & 33 & $29,73 \%$ & 93 & $30,59 \%$ & 202 & $29,88 \%$ \\
\hline Light dig. & 0 & $0,00 \%$ & 63 & $79,75 \%$ & 22 & $66,67 \%$ & 86 & $92,47 \%$ & 176 & $87,13 \%$ \\
\hline Moderate dig. & 0 & $0,00 \%$ & 12 & $15,19 \%$ & 10 & $30,30 \%$ & 5 & $5,38 \%$ & 19 & $9,41 \%$ \\
\hline Heavy dig. & 0 & $0,00 \%$ & 4 & $5,06 \%$ & 1 & $3,03 \%$ & 2 & $2,15 \%$ & 7 & $3,47 \%$ \\
\hline TOTAL NISP & 26 & $100,00 \%$ & 348 & $100,00 \%$ & 111 & $100,00 \%$ & 304 & $100,00 \%$ & 676 & $100,00 \%$ \\
\hline
\end{tabular}

Tabla 3: Presencia de procesos digestivos (NISP) en las especies más relevantes de Pié Lombard. / Presence of digestion processes (NISP) on more relevant species in Pié Lombard. 
$(12,99 \%, n=256)$ y marcas de raíces $(11,98 \%, n=236)$ en un buen número de restos. Menos comunes son las impregnaciones de manganeso $(7,87 \%, n=155)$, los huesos fisurados $(6,60 \%, n=130)$ y las oxidaciones $(3,35 \%, n=66)$.

En otro sentido, analizamos las fracturas cuando fue posible. En términos generales, un 25,89\% $(n=510)$ de los huesos estaban enteros, frente a un $74,11 \%$ $(n=1460)$ fragmentados. Los escasos restos de Alectoris graeca eran los más completos en nuestra muestra.

Los huesos analizados y clasificados siguiendo el criterio de Villa y Mahieu (1991) dieron los siguientes resultados: por delineación, la mayoría de los restos estudiados tenían una fractura curva $(69,22 \%, n=551)$. Algo menos de una tercera parte, en cambio, poseían una delineación transversal $(30,78 \%, n=245)$. La mayoría de los ángulos de fractura eran mixtos $(69,48 \%, n=551)$, aunque también encontramos rectos $(19,29 \%, n=153)$ y oblicuos $(11,22 \%, n=89)$. Por último, la mayoría de las texturas eran dentadas $(57,99 \%, n=461)$, seguidas de cerca por un buen porcentaje de suaves $(42,01 \%, n=334)$.

Por otro lado, nuestra tipología morfológica (Romero et al., 2016a) muestra la siguiente frecuencia de tipos (Tabla IV). Primero, los tipos curvos son los más abundantes. En segundo lugar, el tipo mixto TC posee una buena representación. El tipo transversal más habitual es el T1. El resto de tipos poseen representaciones minoritarias.

Entre diferentes grupos de aves, la presencia de esta tipología morfológica es similar exceptuando en los casos de C3 y TC. En Alectoris graeca y Columba livia existen un $22,44 \%(n=46)$ de los huesos con fracturas de tipo C3, un $9,27 \%(n=19)$ con TC y un $68,29 \%$ $(n=140)$ con otros tipos. No obstante, en el género Pyrrhocorax hay un $15,40 \%(n=83)$ de los restos con un tipo C3, un $15,96 \%(n=86)$ con TC y un $68,65 \%(n=370)$ con otros tipos. Esta distribución es estadísticamente significativa $(X 2=8.954 ; p=0.0113)$.

\section{DISCUSIÓN}

Las especies dominantes son las pertenecientes al género Pyrrhocorax (Pyrrhocorax pyrrhocorax y Pyrrhocorax graculus). Las chovas suelen estar presentes en un gran número de yacimientos pleistocenos en Europa Occidental (Sánchez-Marco, 1996, 2004), así que debieron ser animales muy comunes. Su presencia no parece verse limitada a zonas de altura, como es el caso de Pyrrhocorax graculus en la actualidad. Tras las chovas, la fuerte presencia de Columba livia (junto con la existencia puntual de restos de Aquila chrysaetos y de aves de la familia Hirundidae) indica una zona de roquedo en los alrededores de Pié Lombard. Además, tanto el género Pyrrhocorax como especies como Alectoris graeca y Perdix perdix son típicas de áreas abiertas. Sin embargo, Coccothraustes coccothraustes y Turdus merula ponen de manifiesto la existencia de algunas masas boscosas, quizás a alguna distancia del sitio. Además, la presencia puntual de aves acuáticas (Anas platyrhynchos) indica una proximidad relativa de zonas de agua. Por otro lado, las especies presentes revelan un clima templado (Sánchez-Marco, 1996).

En términos generales, la representación anatómica (Figura 1) está dominada por huesos largos, siendo especialmente abundantes los de las alas. En segundo lugar, también los huesos de las patas poseen una buena representación, sobre todo los distales. Los huesos del tronco están menos representados, aún apareciendo un buen número de coracoides y escápulas. Generalmente, esta representación no coincide con ninguno de los modelos unicausales propuestos por algunos autores (p. e., Mourer-Chauviré, 1983).

\begin{tabular}{|c|c|c|c|c|c|c|c|c|c|c|c|c|}
\hline & A. graeca & \%A. graeca & C. livia & \%C. livia & P. pyr. & \%P. pyr. & P. grac. & \%P. grac. & $P$. indet & $\% P$. indet & TOTAL & \%TOTAL \\
\hline C1 & 2 & $10,53 \%$ & 24 & $12,90 \%$ & 8 & $10,67 \%$ & 16 & $12,90 \%$ & 57 & $16,76 \%$ & 107 & $14,38 \%$ \\
\hline C2 & 4 & $21,05 \%$ & 18 & $9,68 \%$ & 9 & $12,00 \%$ & 21 & $16,94 \%$ & 33 & $9,71 \%$ & 85 & $11,42 \%$ \\
\hline $\mathrm{C} 3$ & 5 & $26,32 \%$ & 41 & $22,04 \%$ & 13 & $17,33 \%$ & 21 & $16,94 \%$ & 49 & $14,41 \%$ & 129 & $17,34 \%$ \\
\hline $\mathrm{T} 1$ & 3 & $15,79 \%$ & 28 & $15,05 \%$ & 10 & $13,33 \%$ & 9 & $7,26 \%$ & 40 & $11,76 \%$ & 90 & $12,10 \%$ \\
\hline T2 & 0 & $0,00 \%$ & 16 & $8,60 \%$ & 9 & $12,00 \%$ & 10 & $8,06 \%$ & 29 & $8,53 \%$ & 64 & $8,60 \%$ \\
\hline T3 & 0 & $0,00 \%$ & 6 & $3,23 \%$ & 2 & $2,67 \%$ & 3 & $2,42 \%$ & 13 & $3,82 \%$ & 24 & $3,23 \%$ \\
\hline $\mathrm{T} 4$ & 2 & $10,53 \%$ & 7 & $3,76 \%$ & 4 & $5,33 \%$ & 5 & $4,03 \%$ & 11 & $3,24 \%$ & 29 & $3,90 \%$ \\
\hline TL1 & 1 & $5,26 \%$ & 3 & $1,61 \%$ & 3 & $4,00 \%$ & 6 & $4,84 \%$ & 4 & $1,18 \%$ & 17 & $2,28 \%$ \\
\hline TL2 & 2 & $10,53 \%$ & 7 & $3,76 \%$ & 7 & $9,33 \%$ & 8 & $6,45 \%$ & 20 & $5,88 \%$ & 44 & $5,91 \%$ \\
\hline TL3 & 0 & $0,00 \%$ & 2 & $1,08 \%$ & 1 & $1,33 \%$ & 1 & $0,81 \%$ & 3 & $0,88 \%$ & 7 & $0,94 \%$ \\
\hline $\mathrm{L}$ & 0 & $0,00 \%$ & 15 & $8,06 \%$ & 3 & $4,00 \%$ & 7 & $5,65 \%$ & 18 & $5,29 \%$ & 43 & $5,78 \%$ \\
\hline TC & 0 & $0,00 \%$ & 19 & $10,22 \%$ & 6 & $8,00 \%$ & 17 & $13,71 \%$ & 63 & $18,53 \%$ & 105 & $14,11 \%$ \\
\hline Total & 19 & $100 \%$ & 186 & $100 \%$ & 75 & $100 \%$ & 124 & $100 \%$ & 340 & $100 \%$ & 744 & $100,00 \%$ \\
\hline
\end{tabular}

Tabla 4: Presencia de nuestra tipología morfológica en las especies más comunes del conjunto. / Presence of our morphological typology on more common species in our sample. 
No obstante, sí existen diferencias entre algunos grupos de aves. En los restos de Alectoris graeca y Columba livia los elementos del tronco son más abundantes que los de las patas. Por contra, en el género Pyrrhocorax los restos de las patas son más frecuentes que los del tronco. En este sentido, podemos relacionar una representación anatómica diferencial con la acción diferenciada de determinados agentes (Mourer-Chauviré, 1979, 1980, 1983, Vilette, 1983). Así, la abundancia de carpometacarpos y tarsometatarsos suele estar relacionada con la depredación de las aves por parte de rapaces o incluso con su muerte natural en entornos kársticos. Este parece ser el caso de las especies del género Pyrrhocorax. Por otro lado, la presencia de huesos ricos en contenido muscular, como húmeros o coracoides puede ser sintomática, según algunos trabajos, de la aportación por la caza por parte de humanos (Mourer-Chauviré, 1989, Díez et al., 1995). Este podría ser el caso de Alectoris graeca y Columba livia en Pié Lombard. Adicionalmente, debemos tener en cuenta la posibilidad de la destrucción de algunos elementos anatómicos durante el procesado y el consumo humano, tal y como se ha indicado para otros yacimientos de esta misma cronología (Blasco et al., 2016). En suma, la acumulación aquí presentada posee un origen mixto. En dicho conjunto han influido las preferencias de depredadores humanos y no humanos, quienes han explotado desigualmente las presas disponibles en el entorno.

En este panorama, la intervención humana como agente tafonómico parece ser ocasional. La presencia del fuego es escasa y, cuando está presente, el resultado son superficies quemadas a bajas temperaturas y/o con una baja exposición del hueso, quizás por la presencia de carne en ellos (Figura 2a). Este tipo de fenómenos, con dobles coloraciones, ocurren cuando los huesos son expuestos al fuego con carne (Rosell, 2001, Laroulandie, 2005a). Sin embargo, como en otros depósitos de origen mixto (Blasco et al., 2016), podemos mantener que la acción del fuego y el propio consumo humano pudieron destruir cierto número de elementos anatómicos consumidos. Este parece haber sido el caso de los restos de Alectoris graeca y Columba livia.

Aunque el porcentaje de huesos avianos con marcas de corte puede parecer bajo (2,99\%), es similar al hallado en otros yacimientos del Pleistoceno Superior. Por ejemplo, en Gorham's Cave el porcentaje de huesos de aves (de los géneros Columba, Corvus, Pica y Pyrrhocorax) con marcas de corte es de un 2,44\% para los niveles del Paleolítico Medio y de un 2\% para el Paleolítico Superior (Blasco et al., 2016:67). El porcentaje es mayor en otros sitios, como Cova Negra (Martínez et al., 2016). Pero en este último yacimiento existen bastantes menos restos de aves y podría tratarse de una acumulación realizada exclusivamente por parte de humanos.

Atendiendo a la disposición de las marcas de corte sobre los elementos anatómicos aquí analizados, parece lógico mantener que la prioridad de los grupos neandertales fue la carne de las aves. Así, existe un gran número de incisiones y raspados en radios, ulnas, húmeros, coracoides y escápulas (Figura 2c y d). Estos huesos poseen atractivos paquetes musculares. En suma, raspados oblicuos y longitudinales en radios, ulnas y húmeros hacen hincapié en las labores de extracción de carne (Díez et al., 1995) (Figura 2b).

Sin embargo, existen algunas marcas de elementos distales con bajos índices de carne. Esta cuestión pone de relieve la posible existencia de actividades de extracción de plumas, tal y como se ha planteado en otros sitios: en Gorham's Cave con palomas (Blasco et al., 2016) y en Kaprina, Fumane y Lex Fieux con rapaces (Peresani et al., 2011, Radovcic et al., 2015, Laroulandie et al., 2016). En nuestra opinión, si la extracción de plumas genera incisiones en los huesos es porque el ave es total o parcialmente despellejada. La extracción de plumas ocasional o de forma individual, como un paso necesario para el posterior procesado y/o consumo de la carne, no debería generar marcas de corte. Las referencias experimentales hasta la fecha (Romandini et al., 2016, Pedergnana y Blasco, 2016) coinciden en señalar la presencia de marcas en las zonas distales de las alas como una de las evidencias generadas durante la extracción de plumas. Sin embargo, aún no existen trabajos experimentales centrados en la viabilidad de las labores de despellejado sobre palomas o perdices (o aves de tallas similares) para el posterior uso de las plumas de las alas, o sobre las huellas que éstas generan sobre las carcasas. Por tanto, preferimos señalar a la carne como la principal motivación para la captura y el procesado de las aves, sin poder asegurar la extracción de plumas como otra posible acción (Blasco et al., 2016), si bien estas labores podrían explicar la significativa presencia de huesos de las alas de palomas y perdices, cuya acumulación parece ser efectuada principalmente por parte de humanos.

Por otro lado, las marcas de dientes y las fracturas generadas por las mordeduras parecen pertenecer a pequeños carnívoros en la mayor parte de las ocasiones. Los casos en los cuales hemos hallado peeling son muy esporádicos, así como otros tipos de fracturas derivadas de la actividad humana sobre los huesos durante su desarticulación y consumo (Laroulandie, 2005b). Adicionalmente, la presencia de algunos huesos con trazas de digestión heavy (Figura 3c) puede ser atribuida a la acción de pequeños carnívoros. De acuerdo con los restos de depredadores presentes en el sitio (Gerber, 1973, Texier, 1974), los carnívoros que probablemente intervinieron sobre las carcasas de las aves pudieron ser pequeños cánidos como el zorro o félidos como el gato salvaje y el lince. En terminos generales, los daños derivados de la masticación observados sobre los huesos avianos de Pié Lombard coinciden con los daños generados por el lince sobre aves y pequeñas presas (Rodríguez-Hidalgo et al., 2013, $2015,2016)$. Así, hallamos bordes crenulados en los paños de las fracturas de los huesos largos, pits en las 
superficies corticales de las diáfisis y punctures sobre la epífisis (Figura 3a, b, d y e).

No obstante, existen algunas marcas de dientes sobre huesos con marcas de corte con características similares a otros generados en trabajos experimentales de consumo humano de aves (p. e., Romero et al., 2016a). Este hecho nos permite plantear que algunas marcas de dientes pertenecen muy probablemente a humanos. Este es el caso de algunos pits circulares (en torno a 0,5 mm de eje máximo) sobre superficies corticales de huesos largos (Saladié et al., 2013, Romero et al., 2016b) (Figura 2b). En resumen, no siempre poseemos las herramientas metodológicas para poder discernir claramente entre marcas de tipo primario realizadas por humanos u otros animales (Romero et al., 2016b). Además, el origen mixto del conjunto aviano de Pié Lombard complica aún más este asunto, quizás con la posible actuación de diferentes agentes sobre los mismos elementos.

Mientras que los patrones de digestión más potentes suelen relacionarse con rapaces diurnas o carnívoros terrestres, la mayoría de las trazas de digestión son moderadas o light. Siguiendo la caracterización propuesta por varios autores (Bochenski, 2005, Rufà et al., 2016b), nuestra muestra parece haber sido acumulada principalmente por rapaces nocturnas. De hecho, encontramos similitudes con las acumulaciones generadas por especies como Bubo bubo y Strix aluco: predominancia de elementos de las alas sobre los restos de las patas, abundancia de huesos de las extremidades sobre los del tronco, fracturación en fresco moderada, trazas de digestión light o moderada y abundancia de carpometacarpos, ulnas, húmeros y tarsometatarsos (Rufà et al., 2016b:57). Sin embargo, no existen restos óseos de estos depredadores en Pié Lombard. En esta dirección, podemos considerar la posible intervención de Aquila chrysaetos y Corvus cf. corone en el yacimiento. Además, no podemos olvidar que la ausencia de restos craneales, esternones y quillas, característicos de las acumulaciones de Aquila chrysaetos y rapaces nocturnas (Bochenski et al., 2009) pueden deberse a razones de conservación diferencial.

En relación con las alteraciones diagenéticas, la relativa abundancia de raíces en la superficie completa de varios huesos de aves nos indica la existencia de cobertura vegetal tras el enterramiento. Igualmente, el alto porcentaje de restos con concreciones e impregnaciones de manganeso y óxidos señala altos índices de humedad en el área de enterramiento, por lo que debemos entender que la formación del depósito tuvo lugar en el mismo abrigo kárstico.

Por un lado, la fracturación es alta en todas las especies mayoritarias, en contraste con los escasos restos de Alectoris graeca (cuya contribución parece ser exclusivamente antrópica). La mayoría de los huesos poseen delineaciones curvas, ángulos mixtos y bordes dentados como consecuencia del alto nivel de consumo por parte de los diferentes depredadores. Abundan todos los tipos curvos (C1, C2, C3), relacionados con las actividades de consumo llevadas a cabo sobre los huesos avianos. Suponemos que todos los depredadores fracturan los huesos, en mayor o menor medida. Como ya hemos indicado, parece ser que, en el depósito de Pié Lombard, el consumo humano destruye elementos del tronco de palomas y perdices y genera fracturas de los tipos C1, C2 y C3 sobre los huesos largos, siguiendo paralelos experimentales (Romero et al., 2016a).

Por otro lado, la presencia de fracturas transversales (especialmente del tipo T1, la más habitual) son indicativas de fragmentación en seco, probablemente a partir de alteraciones postdeposicionales, una vez que el conjunto se encuentra enterrado.

En términos generales, todas las especies más numerosas de aves poseen porcentajes similares de tipos de fractura. No obstante, existe una diferencia remarcable en este sentido: el tipo C3 es muy abundante en Columba livia y Alectoris graeca mientras que el tipo TC es muy común sobre las chovas. Ello es indicativo de una remodelación tafonómica del sitio. Consumidos por otros depredadores y con una fractura curvada inicial, algunas fracturaciones postdeposicionales en seco se superponen a las curvadas en los restos de Pyrrhocorax tras el enterramiento, generando tipos TC. Por contra, palomas y perdices debieron ser enterradas en momentos diferentes y durante la ocupación humana del abrigo rocoso. Probablemente este enterramiento ocurrió más rápidamente, con menor fragmentación diagenética y preservando con mayor asiduidad las fracturas curvas acaecidas en fresco.

\section{CONCLUSIONES}

El sitio de Pié Lombard ha proporcionado un rico repertorio tecnológico adscrito al Musteriense y abundantes restos faunísticos del Pleistoceno Superior. La acumulación ósea de fauna posee un origen mixto de las diferentes especies que la conforman (incluyendo mamíferos y aves).

La colección de restos avianos de Pié Lombard contiene 1970 NR (1680 NISP). Este conjunto está dominado, fundamentalmente, por chovas y palomas. Las especies presentes indican un hábitat de roquedo, espacios abiertos y una temperatura templada.

El conjunto óseo de aves aquí estudiado posee un significativo grado de fracturación como resultado de varios procesos de consumo de diferentes depredadores (humanos, rapaces y pequeños carnívoros). Después del enterramiento, la fragmentación fue especialmente importante sobre los restos de chovas, acumuladas cuando las comunidades neandertales estaban ausentes en el abrigo rocoso. El análisis tafonómico indica la existencia de cobertura vegetal sobre el depósito, así como que éste se formó en el propio entorno kárstico. 
La colección de restos avianos es el resultado de una acumulación de origen mixto en la cual han intervenido diversos agentes tafonómicos. Las aves rapaces transportaron a sus presas al abrigo rocoso, donde las consumieron y luego regurgitaron sus restos. Las trazas de digestión leves o moderadas sobre la mayoría de los fragmentos y la abundancia de carpometacarpos, ulnas, húmeros y tarsosmetatarsos indican que las rapaces nocturnas poseen un rol relevante, aparte de la posible intervención de algunas rapaces diurnas. En segundo lugar, algunos carnívoros (entre los que probablemente se encontraba un pequeño félido) aportaron algunas aves cazadas.

Por último, las sociedades neandertales frecuentaron habitualmente el abrigo rocoso, capturando, procesando y consumiendo palomas, perdices y, ocasionalmente, algunas chovas. Estos pájaros fueron cazados en el entorno más inmediato del sitio. La intervención humana sobre las carcasas es identificada por numerosas marcas de corte en los huesos, con predominio de actividades de descarnado, fracturas en fresco (generalmente curvadas, de ángulos mixtos e irregulares) y huellas de fuego. Sin excluir una posible extracción y uso de las plumas, las comunidades neandertales de Pié Lombard cazaron a las aves por su carne, complementando así las proteínas y grasas obtenidas a partir de la caza de mamíferos. En este sentido, resulta interesante establecer una posible relación entre la explotación de aves y la presencia de restos humanos infantiles. Las comunidades neandertales parecen ser de pequeño tamaño, abarcando un grupo familiar en sentido extenso, dentro del cual los miembros más jóvenes pudieron desarrollar las actividades de captura de pequeñas presas.

Así, Pié Lombard es un yacimiento en el cual el small game fue parte real de la dieta. Este sitio puede incluirse, junto con otros de Europa Occidental (p. e., Cochard et al., 2012, Blasco et al., 2016), en la lista de yacimientos en los cuales se demuestra la capacidad de las sociedades neandertales para capturar, procesar y consumir aves como parte de una estrategia económica continuada en el espacio y en el tiempo.

\section{AGRADECIMIENTOS}

Nuestra más sincera gratitud a Pierre-Jean Texier, Cécile Mourer-Chauviré y Emmanuel Desclaux por su ayuda y disposición. Sin su colaboración, este trabajo no habría sido posible. Además, nos gustaría dar las gracias a Antonio Sánchez-Marco, Ruth Blasco y Álvaro Arrizabalaga por sus comentarios y apoyo a la hora de preparar este trabajo. A. J. R. es parte del grupo de investigación en Prehistoria de la Universidad del País Vasco (UPV/EHU) (IT-622-13) y tiene un contrato como Personal Docente e Investigador en Formación (PDIF) de la Universidad del País Vasco/Euskal Herriko Unibertsitatea (UPV/EHU).

\section{BIBLIOGRAFIA}

Andrews, P., 1995. Experiments in Taphonomy. Journal of Archaeological Science 22, 147-153.

Bailey, G., 2007. Time perspectives, palimpsests and the archaeology of time. Journal of Anthropological Archaeology 26, 198-223.

Bochenski, Z.M., 2005. Owls, diurnal raptors and humans: signatures on avian bones. In: O'Connor T (ed.), Biosphere to Lithospere. New Studies in Vertebrate Taphonomy, 31-45. Oxbow Books, Oxford.

Bochenski, Z.M., Nekrasov, A.E., 2001. The taphonomy of Sub-Atlantic bird remains from Bazhukovo III, Ural Mountains, Russia. Acta Zoologica Cracoviensia 44(2), 93-106.

Bochenski, Z.M., Tomek, T., Tornberg, R., Wertz, K., 2009. Distinguishing nonhuman predation on birds: pattern of damage done by the white-tailed eagle Haliaetus albicilla, with comments on the punctures made by the golden eagle Aquila chrysaetos. Journal of Archaeological Science 36, 122-129.

Blasco, R., Rosell, J., Rufà, A., Sánchez-Marco, A., Finlayson, C., 2016. Pigeons and choughs, a usual resource for the Neanderthals in Gibraltar. Quaternary International, 421, 62-77.

Bramwell, D., Yalden, W., Yalde, P.E., 1987. Black grouse as the prey of the golden eagle at an archaeological site. Journal of Archaeological Science 14, 195-200.

Cassoli, P.F., Tagliacozzo, A., 1997. Butchering and cooking of birds in the Palaeolithic site of Grotta Romanelli (Italy). International Journal of Osteoarchaeology 7, 303-320.

Cochard, D., Brugal, J.P., Morin, E., Meignen, L., 2012. Evidence of small fast game exploitation in the Middle Palaeolithic of Les Canalettes Aveyron, France. Quaternary International 264, 32-51.

Díez, J.C., Sánchez-Marco, A., Moreno-Lara, V., 1995. Grupos avicaptores del Tardiglaciar: Las aves de Berroberria. Munibe Antropologia-Arkeologia 47, 3-22.

Eastham, A., 1998. Magdalenians and Snowy Owls: bones recovered at the Grotte de Bourrouilla (Arancou, Pyrénées Atlantiques). Paléo 10, 95-107.

Fa, J.E., Stewart, J.R., Lloveras, L., Vargas, J.M., 2013. Rabbits and hominin survival in Iberia. Journal of Human Evolution 64, 233-241

Gerber, J.-P., 1973. La faune des grands mammifères du Würm ancien dans le Sud-Est de la France. Université de Provence, Ph. D. dissertation.

Landt, M.J., 2007. Tooth marks and human consumption: ethnoarchaeological mastication research among foragers of the Central African Republic. Journal of Archaeological Science 34, 1629-1640.

Laroulandie, V., 2003. Exploitation des Oiseaux au Magdalénien en France: Etat des lieux. In: Costamagno S, Laroulandie $\checkmark$ (eds.), Mode de vie au Magdalénien: Aports de l'archéozoologie. Actes du colloque 6.4 du XIVe Congrés du l'UISPP, Liége, Belgique, 2-8 septembre 2001, 129-138, Archaeopress, British Archaeological Reports 1114.

Laroulandie, V., 2005a. Bird exploitation pattern: the case of Ptarningan Lagopus sp. In the Upper Magdalenian site of La Vache (Ardiège, France). In: Grupe, G., Peters, J. (eds.), Feathers, Grid and Symbolism Birds and Humans in the Ancient Old and New Worlds. Proceedings of the 5th. Meeting of the ICAZ, 165-178, Munich.

Laroulandie, V., 2005b. Anthropogenic versus non-anthropogenic bird bone assemblages: new criteria for their distinction. In: In: O'Connor T (ed.), Biosphere to Lithospere. New Studies in Vertebrate Taphonomy, 25-30. Oxbow Books, Oxford. 
Laroulandie, V., Faivre, J.P., Gerbe, M., Mourre, V., 2016. Who brought the bird remains to the Middle Palaeolithic site of Les Fieux (Southwestern, France)? Direct evidence of a complex story. Quaternary International 421, 116-133.

Lloveras, L., Moreno-García, M., Nadal, J., 2008. Taphonomic study of leporid remains accumulated by the Spanish Imperial Eagle (Aquila adalberti). Geobios 41, 91-100.

Lloveras, L., Moreno-García, M., Nadal, J., 2009. Butchery, cooking and human consumption marks on rabbit (Oryctolagus cuniculus) bones: an experimental study. Journal of Taphonomy 7, 179-201.

Lloveras, L., Moreno-García, M., Nadal, J., 2012. Assessing the variability in taphonomic studies of modern leporid remains from Eagle Owl (Bubo bubo) nest assemblages: the importance of age of prey. Journal of Archaeological Science 39, 37543764 .

Lyman, R.J., 1994. Vertebrate Taphonomy. Cambridge University Press, Cambridge.

Lyman R.J., 2008. Quantitative Paleozoology. Cambridge University Press, Cambridge.

Martínez, G., 2009. Human chewing bone surface modification and processing of small and medium prey amongst the Nukak (foragers of the Colombian Amazon). Journal of Taphonomy 7, 1-20

Mourer-Chauviré, C., 1975. Les oiseaux du Pléistocène moye et supérieur de France. Université Claude-Bernard Lyon, Ph. D. dissertation.

Mourer-Chauviré, C., 1979. La chasse aux oiseaux pendant la préhistoire. La Recherche 106, 1202-1210.

Mourer-Chauviré, C., 1980. Las aves del sitio de ocupación achelense de Áridos-I (Arganda, Madrid). In: Santonja, M., López, N., Pérez, A., (coords.), Ocupaciones achelenses en el valle del Jarama, 145-160. Diputación Provincial de Madrid, Madrid.

Mourer-Chauviré, C., 1983. Les oisseaux dans les habitats paléolithiques: gibier des hommes ou proies des rapaces?. In: Grigson C, Clutton-Brock J. (eds.), Animals and Archaeology: 2. Shell, Middens, Fishes and birds, 111-124. B.A.R. (International Series), 183

Negro, J.J., Blasco, R., Rosell, J., Finlayson, C., 2016. Potential exploitation of avian resources by fossil hominins: An overview from ethnographic and historical data. Quaternary International 421, 6-11.

Nicholson, R.A., 1993. A Morphological Investigation of Burnt Animal Bone and an Evaluation of its Utility in Archaeology. Journal of Archaeological Science 20, 411-428.

Noe-Nygaard, N., 1989. Man-made trace fossils on bones. Human Evolution 4, 461-491.

Pedergnana, A., Blasco, R., 2016. Characterising the exploitation of avian resources: An experimental combination of lithic use-wear, residue and taphonomic analyses. Quaternary International 421, 255-269.

Porraz, G., 2005. En marge du milieu alpin. Dynamiques de formation des ensembles lithiques et modes d'occupation des territoires au Paléolithique moyen. Université de Provence, Ph. D. dissertation

Rodríguez-Hidalgo, A., Saladié, P., Marín, J., Canals, A., 2015 Expansion of the referential framework for the rabbit fossil accumulations generated by Iberian lynx. Palaeogeography, $\mathrm{Pa}$ laeoclimatology, Palaeoecology 418, 1-11.

Rodríguez-Hidalgo, A., Saladié, P., Marín, J., Canals, A., 2016. Bird-bone modifications by lberian lynx: A taphonomic analysis of non-ingested red-legged partridge remains. Quaternary International 421, 228-238.
Rodríguez-Hidalgo, A., Lloveras, L., Moreno-García, M., Saladié, P., Canals, A., Nadal, J., 2013. Feeding behaviour and taphonomic characterization of non-ingested rabbit remians produced by the Iberian Iynx (Lynx pardinus). Journal of Archaeological Science 40, 3031-3045.

Romandini, M., Fiore, I., Gala, M., Cestari, M., Guida, G., Tagliacozzo, A., Peresani, M., 2016. Neanderthal scraping and manual handling of raptors wing bones: Evidence from Fumane Cave. Experimental activities and comparison. Quaternary International 421, 154-172.

Romero, A.J., Díez, J.C., Rodríguez, L., Arceredillo, D., 2016a. Anthropic fractures and human tooth marks: An experimental approach to non-technological human action on avian long bones. Quaternary International 421, 219-227.

Romero, A.J., Díez, J.C., Saladié, P., 2016b. Mammal bone surface alteration during human consumption: An experimental approach. Journal of Archaeological Science: Reports 8, 82-89.

Rosell, J., 2001. Patrons d'aprofitament de les biomases animals durant el Pleistoce Inferior i Mig (Sierra de Atapuerca, Burgos) i Superior (Abric Romani, Barcelona). Universitat Rovira i Virgili, Ph. D. dissertation.

Rufà, A., Blasco, R., Roger, T., Moncel, M.H., 2016a. What is the taphonomic gent responsible for the avian accumulation? An approach from the Middle and Early Late Pleistocene assemblages from Payre and Abri des Pêcheurs (Ardèche, France). Quaternary International 421, 46-61.

Rufà, A., Blasco, R., Rivals, F., Rosell. J., 2016b. Who eats whom? Taphonomic analysis of the avian record from the Middle Paleolithic site of Teixoneres Cave (Moià, Barcelona, Spain). Quaternary International 421, 103-115.

Saladié, P., Rodríguez-Hidalgo, A., Díez, J.C., Martín-Rodríguez, P., Carbonell, E., 2013. Range of bone modifications by human chewing. Journal of Archaeological Science 40, 380-397.

Sánchez-Marco, A., 1996. Aves fósiles del Pleistoceno Ibérico: Rasgos climáticos, ecológicos y zoogeográficos. Ardeola 43, 207-219.

Sánchez-Marco, A., 2004. Avian zoogeographical patterns during the Quaternary in the Mediterranean Region and paleoclimatic interpretation. Ardeola 51, 91-132.

Sanchis, A., 2012. Los lagomorfos del Paleolítico medio en la vertiente mediterránea ibérica. Humanos y otros predadores como agentes de aporte y alteración de los restos óseos en yacimientos arqueológicos. Servicio de Investigación Prehistórica del Museo de Prehistoria de Valencia, Serie de trabajos varios n. 115, Valencia.

Sanchis, A., Fernández-Peris, J., 2008. Procesado y consumo antrópico de conejo en la Cova del Bolomor (Tavernes de la Valldigna, Valencia). El nivel XVIIc (ca 350 ka). Complutum 19, 25-46.

Sanchis, A., Morales, A., Pérez-Ripoll, M., 2011. Creación de un referente experimental para el estudio de las alteraciones causadas por dientes humanos sobre huesos de conejo. In: Morgado, A., Baena, J., García, D. (Eds.), La investigación experimental aplicada a la arqueología. Actas del Segundo Congreso Internacional de Arqueología experimental, 343349, Ronda.

Serjeantson, D., 2009. Birds. Cambridge Manuals in Archaeology, Cambridge.

Shipman, P., Foster, G.F., Schoeninger, M., 1984. Burnt bones and teeth: an experimental study of colour, morphology, crystal structure and shrinkage. Journal of Archaeological Science 11, 307-325.

Stiner, M.C., Kuhn, S.L., 1995. Differential burning, recrystallization, and fragmentation of archaeological bone. Journal of Archaeological Science 22, 223-237. 
Texier, P.-J., 1974. L'industrie moustérienne de l'abri Pié-Lombard (Tourrettes-sur-Loup, Alpes-Maritimes). Bulletin de la Société Préhistorique Française 71, 429-448.

Texier, P.-J., Renault-Miskovsky, J., Dessclaux, E., Lumley, M.A., Porraz, G., Tomasso, A., 2011. L'abri Pié Lombard à Tourrettes-sur-Loup (Alpes-Maritimes): anciennes fouilles (19711985), nouvelles données. Bulletin du Musée d'Anthropologie Préhistorique de Monaco 51, 19-49.

Val, A., De la Peña, P., Wadley, L., 2016. Direct evidence for human exploitation of birds in the Middle Stone Age of South Africa: The example of Sibudu Cave, KwaZulu-Natal. Journal of Human Evolution 99, 107-123.

Valladas, H., Chadelle, J.-P., Geneste, J.-M., Joron, J.-L., Meignen, L., Texier, P.-J., 1987. Datations par la thermoluminescence de gisements moustériens du sud de la France. L'Anthropologie 91, 211-226.
Vaquero, M., 2008. The history of Stone: behavioural inferences and temporal resolution of an archaeological assemblage from the Middle Palaeolithic. Journal of Archaeological Science 35, 3178-3185.

Villa, P., Mahieu, E., 1991. Breakage patterns of human long bones. Journal of Human Evolution 21, 27-48.

Vilette, P., 1983. Avifaunes du Pléistocène final et de l'Holocène dans le Sud de la France et en Catalogne. Atacina, Carcassonne.

Yokoyama, Y., Quaegebeu, J.-P., Bibron, R., Léger, C., 1983. ESR dating of stalagmites of the Caune de l'Arago, the Grotte du Lazaret, the Grotte du Vallonnet and the abri Pié Lombard: a comparison with the U-Th method. Third specialist seminar on TL and ESR dating, Helsingør, Denmark, 1982, 9(part II), 381-389, Helsingør. 